\begin{tabular}{|c|c|}
\hline Citation & $\begin{array}{l}\text { Kim Loyens, (2013) } \\
\text { Grid-group cultural theory and the causal mechanism approach as } \\
\text { requisite partners. Explaining enforcement decisions in a West-European } \\
\text { labour inspection } \\
\text { Innovation: The European Journal of Social Science Research, 26(4), 416- } \\
435 \text {. }\end{array}$ \\
\hline Archived version & $\begin{array}{l}\text { Author manuscript: the content is identical to the content of the published } \\
\text { paper, but without the final typesetting by the publisher }\end{array}$ \\
\hline Published version & http://dx.doi.org/10.1080/13511610.2013.823348 \\
\hline Journal homepage & http://www.tandfonline.com/toc/ciej20/current\#.VCV0zE0cTg8. \\
\hline Author affiliation & $\begin{array}{l}\text { Kim Loyens, Leuven Instititute of Criminology, Faculty of Law, KU Leuven } \\
\text { (University of Leuven), Belgium }\end{array}$ \\
\hline Author contact & $\frac{\text { kim.loyens@law.kuleuven.be }}{+32 \text { (0) } 16329452}$ \\
\hline IR & https://lirias.kuleuven.be/handle/123456789/395421 \\
\hline
\end{tabular}

(article begins on next page) 


\title{
Grid-group cultural theory and the causal mechanism approach as requisite partners. Explaining enforcement decisions in a West- European labour inspection
}

\begin{abstract}
As street-level bureaucrats, labour inspectors enjoy much autonomy and discretion in the performance of their job. To avoid that this freedom is abused, two measures are often taken. First, supervisors keep oversight by inspecting their case files. Second, labour inspectors are subjected to a competitive system of targets that encourages competition. These two measures resemble two subtypes of organisational control in grid-group cultural theory, respectively hierarchical and individualistic control. The impact of both types of control has been analysed in an ethnographic case study in four field organisations of a West-European labour inspection. The overarching grid-group cultural theory framework was particularly valuable to illustrate how tensions between hierarchical and individualistic control lead to inconsistencies in the enforcement styles labour inspectors apply. However, it did not seem sufficient to explain why labour inspectors apply a hierarchical enforcement style in some investigations and an individualistic enforcement style in other investigations. This paper illustrates how the integration of grid-group cultural theory with two middle-range theories in the causal mechanism approach (i.e. moral disengagement and role strain theory) could provide such proximate explanations and thus strengthen the exploratory power of grid-group cultural theory.
\end{abstract}




\section{Introduction}

As typical street-level bureaucrats, labour inspectors enjoy much autonomy in the performance of their job (Lipsky, 1980). This results from the fact that they often conduct inspections on their own, keep their own agenda and perform administrative tasks at home. Hence, their supervisors are given little opportunity to supervise them. In addition, labour inspectors often have wide discretionary powers. These allow them to make a "choice among possible courses of action and inaction" (Davis, 1969: 4). According to some scholars, discretion of street-level bureaucrats is a necessary evil that should be restricted as much as possible (Thompson, 1975; Sabatier \& Mazmanian, 1979; Brigham \& Brown, 1980; Edwards, 1980; Linder \& Peters, 1987). They plead for procedural constraints in the exercise of discretion. Other scholars, however, think of discretion as 'inevitable' (Jowell, 1973), a necessary element in tempering law with humanity (Pollock, 1994) or an essential condition for efficient and effective implementation of rules and policy (Deutsch, 1985; Lincoln, 1985; Rogers \& Joung-Imkim, 1985; Handler, 1986; Bakker \& van Waarden, 1999). Still, they admit that discretionary powers can (and will) be abused. Therefore, the exercise of discretion should be managed properly, for example, through direct supervision, standardization of working procedures, performanceoriented reward systems or attempts to change the organizational culture.

To avoid that labour inspectors abuse the freedom they are granted, supervisors mostly try to steer them with two types of measures: keeping hierarchical oversight and setting quantifiable targets. These two types of measures resemble two subtypes of organisational control in grid-group cultural theory (GGCT), respectively hierarchical and individualistic control (Hood, 1998). The impact of both types of control on labour inspectors' enforcement decisions has been analysed in a case study in four field organisations of a labour inspection in a West-European country. This study was aimed at analysing and explaining how labour inspectors enforce rules in specific investigations. GGCT was used as the main theoretical framework. This theory was particularly valuable to illustrate how tensions between hierarchical and individualistic control measures lead to inconsistencies in the enforcement styles labour inspectors apply in practice. However, it did not seem to be sufficient to explain decisionmaking of labour inspectors in specific situations or, in other words, why labour inspectors apply a hierarchical enforcement style in some investigations and an individualistic enforcement style in other investigations. Therefore, GGCT was supplemented with two middle-range theories (i.e. moral disengagement theory and role strain theory) that identify causal mechanisms, which are able to provide proximate explanations of labour inspectors' decision-making in specific investigations.

\section{Grid-group cultural theory and causal mechanisms as partners}

\section{Grid-group cultural theory: the basics}

GGCT was originally developed in anthropology (Douglas, 1982), but subsequently applied in other research domains (Mars, 1982; Jensen, 1998; Thompson, Ellis, \& Wildavsky, 1990; Hendriks, 1999; Maesschalck, 2004a; Loyens, 2012a; Loyens, 2013). In this paper, Hood (1998)'s application of GGCT on public management will be used. He states that the combination of the two dimensions 'grid' (i.e. stratification) and 'group' (i.e. collectivity) leads to four control types: oversight (high grid, high group), competition (low grid, low group), mutuality (low grid, high group) and contrived randomness (high grid, low group). Because each control type separately leads to 'excesses' (e.g. oversight to rule fetishism) a combination of the four types is needed for a managerial system to be effective. 
GGCT is not only a typology, but also a theory with causal claims. For this paper, three causal claims or causal propositions will be identified. First, the compatibility proposition states that the four cultural types shape the behaviour and values of staff members or, in other words, "[a]s people organise, so they will behave" (Thompson et al., 1990: 97). This central hypothesis of GGCT means that staff members' behavioural style is influenced by organisational factors. Applied to the topic of this paper, hierarchical and individualistic control measures will, respectively, lead to hierarchical and individualistic enforcement styles. In this study, the hierarchical enforcement style ${ }^{1}$ refers to labour inspectors taking certain decisions because they want to follow orders, rules and procedures that they consider just and legitimate, and because they consider it their role and responsibility to follow instructions from superiors. In the individualistic enforcement style, labour inspectors take decisions in a more opportunistic way, strategically aimed at their own personal benefits. Labour inspectors who apply this enforcement style use their discretion to their own advantage, which sometimes encourages them to bend rules and sacrifice due process in order to get results.

Second, the requisite variety proposition implies that the four cultural types are in constant competition with each other for dominance, but each type needs the other "to define itself against" (Maesschalck, 2004b: 31). In other words, the four types are "self-defined adversarially" (Douglas \& Ney, 1998: 104). As a result, "all four [cultural] types are at least potentially and usually actually, always present" (Maesschalck, 2004b: 31) in a given social unit.

Third, the socio-cultural viability proposition $(6,2003)$ states that each cultural type has its own builtin weaknesses or blind-spots that, taken separately, might lead to negative consequences or undesirable excesses (Hood, 1998). Hierarchy could, for example, lead to rule-fetishism and individualism to anarchy (Schwartz, 1991: 765). Only by combining them in a settlement, these weaknesses can be compensated for by the strengths of other types as necessary voices in the discussion (Pollock, Lilie, \& Vittes, 1993; Thompson \& Rayner, 1998). Applied to the topic of this paper, hierarchical and individualistic control measures should be combined to avoid excesses that would result from emphasizing only one of them.

As a result of the requisite variety proposition, the four GGCT control types should always be present in an organization. The socio-cultural viability proposition suggests that there is constant tension between the four types to avoid excesses. However, GGCT fails to explain how staff members deal with this tension in particular situations, and thus fails to explain which cultural type has an impact in particular situations. In other words, while GGCT can give fundamental and distal explanations for enforcement decisions, it is not suitable to provide proximate explanations for behaviour in specific situations or to explain why labour inspectors in some cases apply a hierarchical enforcement style and in other cases an individualistic enforcement style. For the latter types of explanation, it is essential to gain insight into the black box between independent and dependent variables (respectively, control type and enforcement style). For this, inspiration will be drawn from middlerange theories in the causal mechanism approach.

\section{The causal mechanism approach}

\footnotetext{
${ }^{1}$ This paper focuses on enforcement styles and not on particular enforcement decisions of individual labour inspectors, because it aims at finding patterns on the organizational and not on the individual level.
} 
The fundamental ambition of the causal mechanism approach is to provide insight into the black box between two variables of which the correlation has been determined. One not only attempts to specify that a relationship exists, but one also aims to examine exactly why and how this relationship exists (Hedström \& Swedberg, 1998; Mayntz, 2004) by identifying the causal mechanisms and the situational conditions under which they occur. The concept 'causal mechanism' can be defined in multiple ways (see e.g. Stinchcombe, 1991; Hedström, 2005). The author, however, follows the approach of George and Bennett who define causal mechanisms in the tradition of scientific realism as:
[...] ultimately unobservable physical, social, or psychological processes through which agents with causal capacities operate, but only in specific contexts or conditions, to transfer energy, information, or matter to other entities. In so doing, the causal agent changes the affected entity's characteristics, capacities, or propensities in ways that persist until subsequent causal mechanisms act upon it. (George \& Bennett, 2005: 137)

The causal mechanism approach is compatible with GGCT in the sense that two types of causal mechanisms have already been identified in this tradition: positive and negative feedback $(6,2003$ : 396). Positive and negative feedback mechanisms are derived from systems theory, where they constitute two basic forces (Jervis, 1997) that explain how a system can be maintained. Positive feedback is a process in which, more or less like a virtuous circle, a phenomenon's characteristics are constantly being reinforced, for example bureaucratic organisations that become more and more bureaucratic and thus narrow-minded by proliferating rules and procedures $(6,2003: 399)$. Typical for positive feedback is that ultimately a phenomenon radicalizes itself. This could, according to systems theory, in the end even lead to the destruction of the system in which the organisation's capacity to perform its core function is eroded. Therefore, positive feedback should be balanced with negative feedback mechanisms, where one force resists the other $(6,2003: 399)$. Negative feedback is "transformational [...] which indicates that the system is deviating from a prescribed course and should readjust to a new steady state" (Kast \& Rosenzweig, 1972: 450). Applied to GGCT, this means that other cultural types are brought in to control for excesses that could result from positive feedback (e.g. hierarchical measures to control for unrestrained individualism or room for individualistic decision-making to compensate for extremely hierarchical control measures). Uncontrolled negative feedback can, however, become vicious and destructive as well. Hence, both positive and negative feedback mechanisms are needed to keep the system balanced. Perri 6 explains it as follows:

[...] institutional viability must consist in the attempt to institutionalize some kind or kinds of settlement between the forces of positive and negative feedback, which gives them some space to operate (social systems are inherently dynamic and in disequilibrium, and cannot be made static), but which moderates both the violence of their own oscillations and of their destructive dynamics and of their encounters with each other. $(6,2003: 402)$

These positive and negative feedback mechanisms raise GGCT from a mere taxonomy to being a dynamic theory that explains how organisational factors interact by constantly changing positions (Verweij et al., 2006: 821). These feedback dynamics are, however, situated at the macro level, because the analytic focus is on long-term evolutions of organisational systems that do not change overnight (Thompson et al., 1990: 77-78). Therefore, they cannot explain in specific situations how certain organisational factors have an impact on the behavioural style staff members apply. In other 
words, GGCT does not seem to succeed in specifying the precise and proximate causal relationships between organisational factors and enforcement style. This is probably due to GGCT's comprehensiveness, because "the more comprehensive a theory the less successful it is at explaining specific situations" (O'Riordan \& Jordan, 1999: 91). Hence, inspiration will be drawn from middlerange theories in psychology and sociology, which identify causal mechanisms that can be used to explain how certain control measures lead to particular enforcement decisions in specific situations.

\section{Causal mechanisms in two middle-range theories}

The first middle-range theory that will be applied in this study is Bandura (1990a; 1999)'s theory of moral disengagement. The basic argument is that individuals use cognitive mechanisms to reframe their behaviour in ways that downplay their ethical content or moral implications. While the theory was originally developed to explain why people are able to engage in inhumane conduct without apparent distress (e.g. political and military violence), it also operates in individuals' mundane, everyday decision-making (Bandura, 1990b: 43). An important assumption of moral disengagement theory is that, even though the mechanisms it proposes operate at the individual level, they are not considered stable personality traits. Rather, moral disengagement is conceptualized as a cognitive orientation to the world that is influenced by social contexts in an interactive relationship between personal and social influences (Bandura, 2002). Sometimes situational aspects or organisational factors can even 'override' individual values and compel people to engage in actions they would otherwise not take part in (Moore, 2008: 131).

Three mechanisms of moral disengagement are relevant in this study: moral justification, attribution of blame and displacement of responsibility. First, an act can be made to appear less harmful by using the cognition of moral justification. Detrimental behaviour is then portrayed as being worthy because it serves higher social or moral purposes (Bandura, 1999: 195). This mechanism could, for example, explain that despite hierarchical control measures, labour inspectors choose to break the rules to achieve effectiveness. The individualistic control measures then have more impact on the enforcement style through the mechanism of moral justification. Second, the mechanism of attribution of blame restructures cognitions by portraying oneself as being an innocent person driven to harmful behaviour by provocation. A case in point is hitting a suspect because he has brought it on himself by committing a crime and lying about it. Like in the previous example, hierarchical rules are in this case disregarded to obtain results, which is an opportunistic or individualistic enforcement style. Third, moral disengagement can operate by displacing one's responsibility to individuals with a higher authority who may have given an explicit order or may have implicitly condoned the behaviour (Diener, 1977; Kelman \& Hamilton, 1989). A case in point is disregarding the competitive target system imposed by the head office (see below), because a supervisor does not consider this target system important.

The second middle-range theory that will be applied is role strain theory, which attempts to explain how and under which circumstances individuals deal with differing and sometimes even conflicting expectations or role strain. Role theorists (e.g. Merton, 1957; Goode, 1960; Biddle, 1986) claim that, as in theatre, individuals in organisations perform 'parts' or 'roles' for which 'scripts' were written. Scripts can be described as organisationally given role-demands, consisting of "norms, expectations, taboos, responsibilities and the like [...] associated with a given social position" (Levinson, 1959: 172). These expectations or demands are generated by several sources, which can be identifiable persons 
- for example, supervisors, colleagues, citizens, etc. (Merton, 1957; Peterson \& Smith, 2000) - or less personalized sources (Levinson, 1959; Peterson et al., 2000) - for example, internal guidelines, the law, HRM policy, etc. This combined group of personalized and non-personalized sources of role expectations is the 'role-set'.

The expectations generated in this role-set do, however, not necessarily lead to a coherent 'script'. There may be differences or even contradictions between expectations generated by several sources in the role-set, leading to role strain (Goode, 1960). Interesting in role strain theory is the observation that, despite these tensions, "humans are not incapacitated by role strain" (Sieber, 1974: 568), leading to the question "which processes are operative to counteract these strains and under which circumstances do they (fail to) operate?" (Merton, 1957: 112-113). Several role theorists explicitly described the causal mechanisms that explain how individuals manage to cope with the (sometimes contradictory) expectations in particular situations, and thus explain the way in which role expectations under specific situational conditions impact the actual role behaviour (e.g. Merton, 1957; Goode, 1960; Hall, 1972; Thornton \& Nardi, 1975). In other words, they list causal mechanisms that explain how staff members manage to cope with conflicting demands.

Five mechanisms of dealing with role strain were relevant in this study. First, differences in power and authority can explain why certain role expectations are more influential than others. Merton (Merton, 1957: 113) argues that not all sources in the role-set are equally powerful or can in the same way legitimately assert authority to shape the behaviour of staff members. Hence, the choice between incompatible expectations could partly be explained by the degree to which the person considers others powerful and their expectations legitimate (Gross, Mason, \& McEachern, 1958; Rose, Kahle, \& Shokam, 2000: 439). When this mechanism is operational, head office's competitive target system would be more influential in a particular investigation than supervisors' hierarchical oversight, the former being higher in power and authority than the latter.

However, the influence of the power mechanism can be diminished when staff members' activities are insulated from their observation. Merton (1957: 114-115) explains that when staff members' behaviour cannot be observed by powerful actors, the staff members are less subjected to their expectations. In other words, if the head office fails to observe whether or not targets are met, then the impact of their competitive system might diminish.

Third, certain expectations are sometimes considered more crucial because staff members consider their relationship with those who issue these expectations more important than their relationship with others. A case in point is following one's colleagues' expectations and not the supervisor's expectations, because the relationship with colleagues is considered more important than the relationship with the supervisor. If colleagues endorse the competitive target system, while supervisors do not, this mechanism could explain why the individualistic target system still has a dominant impact on labour inspectors' behaviour.

Fourth, if staff members in an organisation give excessive attention to only a few expectations in the role-set, while the actual role expectations are more diverse or balanced, the mechanism of selection is operational. Staff members in an organisation are then convinced that this selection of expectations is most relevant in the script or that only these expectations exist. Reasons for this selection mechanism to be triggered are mostly related to flaws in the communication process, particularly the lack of (clear) communication about certain expectations in the organisation. If individualistic control 
measures are more clearly communicated in an organization than hierarchical control measures, then this mechanism explains why the former have more impact on front-line officers' behaviour.

Fifth, the mechanism of alteration refers to the fact that certain role expectations are misinterpreted by staff members in an organisation. This is often due to ambiguous communication processes and a lack of transparency about actual expectations (Rose et al., 2000: 440). Labour inspectors' autonomous work context could also give them the false idea that they are not obliged to follow hierarchical orders imposed by the head office that limit their discretion. Their individualistic work context then alters their perception of the relevance of hierarchical control measures.

\section{Towards an integration of GGCT and two middle-range theories}

As explained above, GGCT is useful to give an overall explanation of how tensions between organisational control types lead to a variety of enforcement styles that are applied by labour inspectors. However, it does not seem to be sufficient to explain why in some investigations the hierarchical enforcement style and in other investigations the individualistic enforcement style are applied. In other words, GGCT does not suffice to explain how labour inspectors deal with the tension between hierarchical and individualistic control in particular situations. This black box can, at least partly, be opened by supplementing GGCT with mechanisms of moral disengagement and role strain theory (see figure 1). These mechanisms are triggered by situational conditions and accentuate certain aspects in the professional context of labour inspectors, particularly aspects of the hierarchical or individualistic control type, and by doing so encourage, respectively, hierarchical or individualistic enforcement styles. This will in the next part be illustrated on the basis of a case study in a WestEuropean labour inspection.

-- FIGURE 1 ABOUT HERE -

\section{A case study on labour inspectors' enforcement styles}

The integration of GGCT and the two middle-range theories in the causal mechanism approach, discussed above, has been tested in a case study on labour inspectors' enforcement decisions. This section first presents the methodological choices that have been made in this case study and the settings in which the study has been conducted. Then, it lists the most important empirical findings. Finally, in the discussion, implications of these findings for GGCT will be shown.

\section{Research design}

In this case study, ethnographic research methods were used, such as observation (almost 300 hours), informal conversation and in-depth interviewing (23 respondents during 60 hours) in four field organisations, in two judicial districts ${ }^{2}$, of a West-European labour inspection during 21 months. Ethnography is defined in a loose way as a set of methods, which in its most characteristic form "involves the ethnographer participating [...] in people's daily lives for an extended period of time, watching what happens, listening to what is said, asking questions - in fact collecting whatever data

\footnotetext{
${ }^{2}$ In the first setting (in judicial district A) 12 labor inspectors are employed. The other three settings are smaller units (of approximately five to seven labor inspectors), which are physically located at the same office (in judicial district B). Each of those units inspects a separate geographic region. They also have a separate regional supervisor and will therefore, for this study, be considered separate research settings.
} 
are available to throw light on the issues that are the focus of the research" (Hammersley \& Atkinson, 1995: 1).

During interviews and informal conversations, the labour inspectors were asked to think about difficult enforcement decisions they had to make in different investigations. They were asked to give a detailed narrative of the event. The interviews were recorded and transcribed literally and the informal conversations were summarized in detailed reports. Observations were made to complement the narratives, particularly in cases where the discussed event had occurred while the researcher was present, and to study the organisational context that is assumed to have an impact on the decision-making processes. This resulted in rich data and 'thick description' (Geertz, 1973). The data were analysed with the qualitative software Nvivo using topic coding and analytic coding (Richards \& Morse, 2007: 139-143), after which the technique of process-tracing was applied to identify the causal mechanisms that underlie decision-making (George et al., 2005).

The respondents in this study are labour inspectors who predominantly do inspections on illegal employment, unregistered work violations and the application of legal wage and labour conditions (e.g. employers who failed to pay employees the correct wage for their work). Labour inspectors' discretionary powers allow them to respond to these three kinds of violations in a threefold way. First, they could give a warning, which implies no consequences for the employer. Second, they could offer employers the chance to rectify the violation by paying the employee back the deprived wage or compensations (i.e. rectification amount) or by officially registering the employee and paying the respective social security taxes in a retrospective way. Third, they could make a formal report of the violation after which the public prosecutor at the labour court decides to prosecute the employer or to dismiss the case.

To make these enforcement decisions, labour inspectors are given much autonomy in the sense that they often work at home and conduct inspections on their own, leading to limited supervision. To avoid the abuse of discretion and autonomy, supervisors and the head office take different measures. First, the head office performs oversight, which is central in the hierarchical control type (Hood, 1998), by imposing internal guidelines that reduce labour inspectors' discretion (i.e. 'Administrative Instructions'). Moreover, labour inspectors are required to fill out weekly reports in which all their activities are described in detail and to compose case files that contain all decisions concerning an investigation, both of which are inspected by their supervisor. Second, the head office applies the individualistic control type 'competition' (Hood, 1998) by setting up a system of targets in which labour inspectors are encouraged to reach certain quantifiable goals for which they are highly valued by their supervisors (e.g. number of investigations and total rectification amount). These performance indicators are aimed at stimulating competition between different labour inspectors and at preventing the abuse of autonomy. Some supervisors even show these individual 'scores' during staff meetings in so-called league tables to reach that goal. The rectification amounts are considered particularly important, probably because these are in the best interest of employees. Moreover, the head office highly values these kinds of results, leading to labour inspectors attaching much importance to their personal overview of wages and compensations that have been rectified in their investigations. However, respondents claim that some supervisors also emphasize the number of investigations and therefore encourage them to do many 'simple' investigations and avoid the complex ones to make the numbers look good, even though the complex investigations often involve serious cases of social 
fraud. The latter type of decisions is not discussed in this paper, but illustrates possible excesses of performance management systems (see also Loyens, 2012b).

Before discussing the results, two methodological remarks need to be made. First, this case study is a qualitative, exploratory study in a relatively small number of research settings. The decisions that are analysed are thus not necessarily representative for enforcement decisions labour inspectors in general make. However, the goal of this qualitative study is not generalization to a wider population as in quantitative research, but theory-building by developing hypotheses that can be tested in future research (Smaling, 2003; Yin, 2003). Arguably, the findings could lead to more theoretical insight into enforcement decision-making. Second, to protect the respondents' identity, neither the country where the organisations are located nor individual characteristics (such as age, sex ${ }^{3}$ or years of experience) of the labour inspectors will be mentioned.

\section{Results}

The respondents in this study mention 95 enforcement decisions. The individualistic enforcement style is dominant in all four research settings with approximately $50 \%$ of the cases (i.e. 46 cases). The hierarchical enforcement style follows with almost one third of the cases (i.e. 27 cases). Although there are also a few examples in which labour inspectors apply egalitarian, fatalistic ${ }^{4}$ and hybrid $^{5}$ enforcement styles (respectively three, five and 14), only the decision-making processes in the individualistic and hierarchical enforcement decisions will be discussed and explained below, being the central focus of this paper.

\section{Explaining individualistic enforcement decisions}

Almost half of the enforcement decisions that are discussed in this paper lead to an individualistic enforcement style. Labour inspectors then aim at getting high individual results (which is in their own benefit) without worrying whether their decision is in the public interest, will effectively tackle social fraud or is according to the rules. The respondents mention three different types of individualistic enforcement styles: (1) negotiation, (2) anticipation of dismissal and (3) acting for personal gain. This part illustrates how GGCT fails to explain why individualistic control measures have a dominant impact in these cases, and how the two middle-range theories in this study can provide more understanding of the causal processes underlying these enforcement decisions.

\footnotetext{
${ }^{3}$ The respondents will always be referred to with male pronouns, even when they are women.

${ }^{4}$ The findings in this paper result from a larger doctoral study in which the impact of organizational factors on various dilemma types was analysed. The fatalistic behavioural style was particularly important in decisions to start of stop an investigation. Labour inspectors often decided not to continue a particular investigation because they felt powerless to deal with a particular type of social fraud or because there were too many loopholes in the laws they had to enforce. The low level of fatalistic behavioural styles in this paper can be explained by the fact that these 'early stop' decisions were not included in this contribution.

${ }^{5}$ The hybrid enforcement style refers to an enforcement style in which two GGCT types are combined. The low level of hybrid enforcement styles can be explained by the fact that the researcher aimed at finding as many pure enforcement styles as possible to achieve analytic clarity. This sometimes resulted in splitting up complex decisions with combined enforcement styles in various pure types.
} 


\section{Implicit and explicit negotiation}

Most individualistic enforcement decisions in this study are part of a negotiation with the employer ( 38 out of 46 cases). This negotiation occurs in an explicit ( 20 cases) or an implicit way $(16 \text { cases })^{6}$, but is always aimed at looking for a win-win-situation. In such negotiations, employers want to avoid being prosecuted and having to pay high rectification amounts, while labour inspectors want to increase their individual 'scores' in terms of reports or rectification amounts because they will be highly valued by superiors for reaching certain quantifiable targets.

In most 'implicit negotiation' cases, labour inspectors decide to drop certain charges (and thus not make a report) because the employer is willing to come across by paying his employees back compensations and wages they have been deprived of. In return for paying a rectification amount, employers will thus not be prosecuted. For the labour inspectors, this strategy leads to high rectification amounts on their personal overview, which is valued more by their supervisor and the head office than the number of reports (see above). This strategy is not only applied when it concerns rather small violations, such as the absence of part time labour contracts or internal labour regulations codes, but sometimes also when it concerns more serious violations of social law, such as unregistered work violations and part time employees who work outside their time-table. The 'Administrative Instructions' that are issued by the head office are, however, clear when it comes to serious offenses, in the sense that these should always be followed with a report when detected. Negotiation is then not permitted. Notwithstanding, labour inspectors often disregard these instructions to obtain a high rectification 'score'.

In terms of GGCT, the cases above show a tension between two control types. On the one hand, supervisors and the head office encourage labour inspectors to obtain as much results in terms of rectification amounts, which is an example of the individualistic control type 'competition'. On the other hand, the 'Administrative instructions' state that labour inspectors should never drop charges for serious offenses, such as unregistered work, which is an example of the hierarchical control type 'oversight'. However, GGCT is not able to explain why in the situations discussed above the individualistic control type has more impact on labour inspectors' behaviour than the hierarchical control type. In other words, GGCT does not explain why labour inspectors choose to disregard hierarchical rules in favour of the competitive target system. There is thus a black box between the individualistic control type and the individualistic enforcement style.

The middle-range role strain theory can open this black box by identifying the causal processes that explain the link between control type and enforcement style in these cases. In other words, role strain theory offers the mechanisms that explain why the hierarchical control measures of the head office and supervisors fail to avoid these questionable individualistic enforcement decisions. First, the mechanism of selection is relevant in two research settings, particularly in those settings in which most labour inspectors are unfamiliar with the 'Administrative instructions' (i.e. part of 'oversight'). Because their supervisors do not clearly communicate about head office's rules, labour inspectors in these settings falsely believe that they can use their discretion by negotiating with employers in order

\footnotetext{
${ }^{6}$ The remaining two decisions are special cases in which labour inspectors give up their attempt to negotiate because it will not lead to the desired effects. These special cases will not be discussed in this paper.

7 This offense is rather serious because these employees could as well be employed full time and only be registered as part-time employees, which would be a creative form of tax evasion.
} 
to reach their own targets, which is an opportunistic and thus individualistic strategy. Second, inspectors in the other settings who are aware of the 'Administrative instructions' (e.g. because they have read this document on the Intranet) deliberately disregard them in the discussed cases, as a result of the insulation from observability mechanism. Particularly, these labour inspectors deliberately keep the part time employment violations they drop out of the case file, so their supervisor (who would not agree with them dropping these charges) will never know that they have made this decision. The implicit negotiation is then not 'observable' for the supervisor, making it impossible for him to take action. In other words, even though the supervisor has the formal power and authority (another mechanism in role strain theory) to keep oversight on labour inspectors, the insulation from observability mechanism neutralizes this power. This insulation from observability mechanism is also relevant in other cases, particularly those cases in which labour inspectors drop unregistered work violations while concealing it from colleagues who are present during the inspection. To conclude, while being confronted with both competition and oversight, the latter is in these dilemmas consciously or unconsciously disregarded, which can be explained by two mechanisms of role strain theory, and not by only applying the overarching GGCT.

In the 'explicit negotiation' cases, unregistered work violations are also sometimes dropped (which is not allowed according to the 'Administrative instructions'), because employers show their willingness to rectify certain violations, either by paying their employees deprived wages or compensations or by registering their employers retrospectively. Labour inspectors justify these questionable decisions by claiming that the offer to drop these violations is sometimes essential to persuade employers to rectify other violations. However, their decision to make this offer is also inspired by opportunism in the sense that rectification amounts are important targets for their individual scores. Like in the implicit negotiation cases, there is a tension between the competitive target system ('competition') and the 'Administrative instructions' ('oversight'), but GGCT fails to explain why 'competition' has more impact on labour inspectors' enforcement style than 'oversight'. Arguably, the moral justification mechanism opens the black box between control type and enforcement style. Labour inspectors consider it more beneficial and more in the employees' best interest that wages and compensations are rectified than that reports are made for unregistered work violations, which justifies (in their opinion) the decision to disregard head office's instructions.

\section{Anticipation of dismissal}

The second type of individualistic enforcement style refers to disregarding certain violations because labour inspectors anticipate the likely dismissal of these violations by the public prosecutor or the agency that can impose administrative fines. One inspector in this study explains that when he worked in another region the public prosecutor almost never prosecuted unregistered work violations, leading to those violations being punished with only a small administrative fine. Inspectors in that region, therefore, mostly asked the employers to officially register the employees, because then at least the detection would lead to the situation being corrected. When this particular inspector just started working in one of the research settings in this study, he applied the same logic even though most of his colleagues always made a report for unregistered work violations. However, one day he caught an employer the second time for the same violation because he had only registered the worker retroactively for the day of the inspection. The inspector discussed this with his new colleagues and 
learned that the public prosecutor in this region mostly prosecutes unregistered work violations, so he changed his approach. He, however, adds that if the public prosecutor was to stop prosecuting unregistered work violations, he would return to the previous approach. In other words, if the public prosecutor was to decide to change his prosecution policy, the inspector would probably start breaking the head office's rules, which is the risk of following the rules not because they are the rules but because they lead to beneficial results.

In GGCT terms there is a tension between hierarchy (i.e. following 'Administrative instructions') and individualism (i.e. making decisions in an opportunistic way). Although the labour inspector technically follows the rules, he only does so because it is in his own benefit and he would change his behaviour if the benefit would be absent. GGCT cannot explain why individualism is dominant here, but the middle-range moral disengagement theory can. Particularly, the displacement of responsibility mechanism is relevant in this case. The inspector would excuse for rule breaking, by displacing responsibility to the public prosecutor, who would (in the inspector's opinion) fail to do his duty by dismissing the case.

In another research setting, a similar logic is applied concerning violations linked to the status of family members who work as assistants (as opposed to employees). While this is allowed in one-man businesses, it is not allowed in companies with limited liability or public limited companies, because these cannot have relatives. Hence, the managing director should hire relatives as official employees. When labour inspectors detect these kinds of violations, the family members should officially be considered unregistered workers and labour inspectors should make a report, because that is prescribed by the 'Administrative instructions' (part of the hierarchical control type). However, the public prosecutor only rarely prosecutes these kinds of violations, leading to many labour inspectors deciding not to make a report and thus acting in an opportunistic way. This is an example of an individualistic enforcement style, because one only makes a report when it leads to results, particularly the prosecution of the violation. GGCT cannot explain why the tension between hierarchy and individualism leads to an individualistic enforcement style, but moral disengagement theory can. Particularly, by displacing responsibility to the public prosecutor for not doing his job (in the labour inspectors' opinion), the labour inspector excuses for his individualistic behaviour. Because the total number of reports is considered less important for most supervisors than the total amount of rectifications, labour inspectors sometimes use these kinds of violations in their negotiation process with the employers (see above). Only when the detection occurs during joint inspections (i.e. inspections that are conducted by different inspections), most labour inspectors make a report, because then it counts towards the joint inspection statistics, which are highly valued by the head office.

\section{Acting for personal gain}

In the third individualistic enforcement style, labour inspectors are inspired by self-interest. Only one case will be discussed in detail, because it beautifully illustrates an excess of the individualistic control type. A labour inspector is conducting an investigation at the end of the year in which wages need to be rectified. The 'Administrative instructions' prescribe that a retrospective rectification period of three years should be applied in such investigations. However, the inspector decides to only impose a rectification period of one year, which leads to a much lower rectification amount and thus less money 
for the employees. The reasoning behind this decision is that the inspector would not be able to finish his investigation before the end of the year if he would have to calculate the rectification amount for a period of three years, while it would be feasible if the rectification period was only one year. Finishing the investigation before the end of the year is important because then the rectification amount will be included in the individual inspectors' statistics for that year. However, the supervisor has already told the inspectors in his field organisation repeatedly that they should let the social secretary's office ${ }^{8}$ calculate the rectification amount because it takes too much time to do it themselves. This particular inspector does not agree with that rule, because (in his opinion) social secretary's offices often make mistakes that afterwards need to be corrected by the inspector, leading to loss of time. He therefore takes the rather opportunistic decision to do the calculations himself (albeit for a rectification amount of only one year) because only then he can add the rectification amount to his personal statistics for this particular year.

In GGCT terms this case shows a tension between two hierarchical control measures, being head office's rules and supervisor's orders, and the individualistic target system. However, GGCT does not explain why individualism is dominant here. Role strain theory can fill this gap. Particularly, the mechanism insulation from observability counteracts the power mechanism. In other words, despite the hierarchical power of the head office and the supervisor to limit labour inspectors' discretion, neither of them can in practice observe how the inspector makes this specific decision as a result of the large autonomy labour inspectors enjoy in the performance of their job. The supervisor checks the case file afterwards when the decision has already been made. Hence the impact of the hierarchical measures is diminished by the fact that certain aspects of labour inspectors' work are not directly observable for hierarchical actors.

\section{Explaining hierarchical enforcement decisions}

In 27 cases, the labour inspectors in this study apply a hierarchical enforcement style. These decisions can be divided into three types: (1) following orders from judicial authorities, (2) following orders from supervisors and (3) basing one's decision on fairness and equal treatment. In some of these cases there is a tension between hierarchical and individualistic control measures, which results in the application of hierarchical enforcement styles. We will illustrate how both middle-range theories can explain why 'competition' is less of influence in these cases. In other cases, labour inspectors are confronted with various and even conflicting hierarchical orders. GGCT is not able to explain in these specific situations why individuals choose to follow certain orders and disregard others. The findings of this study show that middle-range theories can provide such proximate explanations.

\section{Following orders from judicial authorities}

The first hierarchical style is basing one's decision on orders from judicial authorities. Two different labour inspectors mention an investigation in which they adapt their decision to what they think the public prosecutor wants. Both investigations are linked to violations concerning deprived wages and compensations. Although the labour inspectors know that trying to rectify these violations would be more beneficial for their personal 'score' (rectification amounts being valued more than the number of reports), they decide to make a report. They do so because the investigations are initiated by the

\footnotetext{
${ }^{8} \mathrm{~A}$ social secretary's office is an organization that can be hired to arrange calculation and payment of wages and other HR activities.
} 
public prosecutor, which leads the labour inspectors to believe that they cannot avoid making a report. This is, however, a false perception that can be explained by the alteration mechanism in role strain theory. Particularly, labour inspectors sometimes believe that their discretionary powers are restricted when investigations are initiated by the public prosecutor, which is not the case. The tension between hierarchical and individualistic control measures can thus sometimes be explained by a false perception. This case illustrates that not only a control measure as such but also the perception of a control measure has an impact on behaviour, an idea which is not explicitly adopted in GGCT.

In some other cases labour inspectors are confronted with conflicting hierarchical orders. As mentioned above, GGCT does not provide the tools to explain why inspectors prefer following one order and disregard the other. In the first case, a labour inspector decides to follow the public prosecutor's request to ask the employer to rectify wages of certain employees, but not of others. Although the instructions of the head office state that all employees should be treated equally, the suggestion of the public prosecutor is followed. This can be explained by the importance mechanism in role strain theory that counteracts the power mechanism. In other words, the labour inspector considers the relationship with the public prosecutor more important than the relationship with the head office, even though the latter is higher in authority than the public prosecutor. The head office is able to formally restrict labour inspectors' discretionary powers, while the public prosecutor is not. However, the labour inspector is aware that he will need the 'goodwill' of the public prosecutor to prosecute violations for which he will make reports in the future and therefore considers this relationship more important than the relationship with the head office.

A similar logic applies in the following case in which a labour inspector makes a report because the public prosecutor asks it, even though he himself wants to give a warning. The role strain mechanism importance explains why this labour inspector decides to allow the public prosecutor to restrict his legal discretion, as illustrated in the following quote:

I never asked myself that question [whether the public prosecutor is legally entitled to give orders]. It is in fact a restriction of our discretionary powers, but public prosecutors claim umm think that we work for them (coughs significantly to indicate that this is not the case), even though we are independent... you know, but [...] I am not planning to get into a tussle with them (laugh). I just do what they ask to avoid trouble.

\section{Following orders from supervisors}

In seven cases, labour inspectors use a hierarchical enforcement style, because they follow orders from their supervisor, even when following them does not lead to the best results. In GGCT, the tension between hierarchical control and individualistic control leads to applying a hierarchical enforcement style. In all these cases, the power mechanism in role strain theory explains why hierarchical control is more influential than individualistic control. In the first case, an inspector imposes a very small rectification amount (i.e. 12 euro) because the inspector thinks the supervisor is going to send the file back if he decides to disregard it. The labour inspector (who is still an intern) does not consider it worthwhile to spend much time in this investigation, because it will not significantly increase his personal 'score'. He explains: 
[...] in my opinion, if you have to send that letter by registered mail, then I don't consider it worthwhile, but I did it anyway. [...] If I could have decided it myself (laugh) I would never have done it, especially because it was just an error and not malicious intent of the employer. [...] But because $X$ is my supervisor, I sent the letter. But when I think of it, I consider the cost for society higher than what has come out of it.

In the second case, the same inspector does not drop certain monetary violations (except for a few insignificant compensations for clothing, etc.), because he knows his supervisor wants all wages and compensations to be rectified, giving him little negotiating room. However, he argues that if he cannot come across with a few concessions, the employer will never agree with paying the rectification amount. The inspectors follow the hierarchical order anyway, because the supervisor is considered an authority that can impose his will (which illustrates the power mechanism in role strain theory). In addition, the hierarchical decision is facilitated by the moral disengagement mechanism of rendering the supervisor responsible for a possible failure of the negotiation.

In the other cases, labour inspectors change their decision concerning unregistered work detections because the supervisor asks for it. The inspectors prefer to impose a period during which the employer can rectify the violations by paying social security taxes for the period the employees have been working on the side, so the inspectors can add these amounts to their statistics. However, their supervisor wants unregistered work violations to be responded with a report at all times and the inspectors comply because the supervisor has, in their opinion, the authority to give orders. The insulation from observability mechanism does not operate here, because the supervisor carefully reads the case files that contain the labour inspectors' decisions.

\section{Fairness and equal treatment}

In the third hierarchical enforcement style labour inspectors disregard orders from hierarchical actors, because they are not considered fair. In GGCT terms, there is a conflict between two hierarchical control types, being the paternalistic attitude of treating all clients in an equal and fair way, on the one hand, and following orders from hierarchical actors, on the other. One specific example will be describe in detail to illustrate this type of hierarchical enforcement style. A labour inspector explains that in a particular investigation, he decided not to make a report for certain violations, even though the public prosecutor asked him to do so. Particularly, the public prosecutor requested him to make a report against the current business manager of a firm which committed certain violations, while previous business managers of this firm who committed the same violations were not prosecuted. The labour inspector disregards this order because he does not consider it fair. GGCT does not explain the dominant impact of the fairness or equal treatment principle, while role strain theory can. Particularly, the importance mechanism leads to the power mechanism being counteracted. In other words, the authority and formal power of the public prosecutor are not considered strong enough to change the inspector's decision, because fair treatment is considered more important.

\section{Discussion}

The findings in this study show that GGCT is useful to illustrate the tensions labour inspectors experience between hierarchical control measures (i.e. 'Administrative instructions' of the head office, oversight by supervisors, etc.) and individualistic control measures (i.e. competitive target system). These tensions sometimes lead to the application of a hierarchical enforcement style in which 
rules are applied because they are considered just and legitimate, or the application of an individualistic enforcement style in which opportunism and personal benefits (e.g. in terms of quantifiable results that increase the personal score) are the central motives. As argued above, the mechanisms of positive and negative feedback in GGCT can explain long-term evolutions or shifts in organizational control systems and enforcement styles. However, GGCT is not able to explain in particular situations why individuals apply a hierarchical or an individualistic enforcement style. In other words, GGCT does not offer proximate explanations for decision making in specific situations, leading to a black box between control type and enforcement style. Middle-range theories can open this black box by providing causal mechanisms that can explain the underling decision making processes between control type and enforcement style. These mechanisms thus explain why in some situations labour inspectors decide to follow the rules, while in other situations they decide to disregard them for individualistic reasons.

For the topic of this study, two middle-range theories proved to be particularly useful to explain decision making processes. First, moral disengagement theory was useful because it is closely related to the general topic of dealing with moral dilemmas, which was the overall theme of this study. Second, role strain theory was useful because it beautifully captures the idea of various and sometimes conflicting expectations with which front-line officers are confronted and between which they have to choose in making decisions on the job. However, for other topics different middle-range theories in sociology, cognitive psychology and adjoining domains might be used to provide black box explanations and thus to supplement the comprehensive, but underspecified GGCT. The middle-range theories should thus not be used to replace GGCT, but merely to strengthen its explanatory power by providing building blocks that can explain how the theory works in particular situations.

\section{Conclusion}

This paper combined GGCT with two middle-range theories to gain insight into the black box between independent and dependent variables. Particularly, two middle range theories (i.e. moral disengagement theory and role strain theory) were used to supplement GGCT and to look for proximate explanations for enforcement decisions. This newly developed conceptual framework, an integration of GGCT and the two middle-range theories, was applied in a case study in a WestEuropean labour inspection to explain when and how hierarchical and individualistic control measures have an impact on labour inspectors' enforcement decisions.

The individualistic enforcement style was mostly part of a negotiation process with employers. Although the practice of negotiation is restricted by hierarchical rules of the head office, labour inspectors sometimes broke these rules to reach higher results (mostly in terms of rectification amounts). This was explained by the alteration or the selection mechanism in role strain theory. Particularly, the 'Administrative Instructions' were, respectively, looked at from an altered perspective or not selected as guiding principles. Orders from the head office and supervisors were sometimes also neglected as a result of the insulation from observability mechanism, which can operate as a result of the high degree of autonomy labour inspectors have and which sometimes even overrules the power mechanism. In some cases, labour inspectors seemed to follow the head office's instructions, but not necessarily because they were considered hierarchical instructions, but because following them led to interesting results for the labour inspector, such as high rectification amounts that count for one's individual statistics. Following the instructions was then considered an 
opportunistic strategy, linked to the head office's pressure for numbers. Although the motive behind following the instructions does not seem to be very relevant as long as the instructions are followed, this opportunistic strategy can be very risky. This is particularly the case when instructions are changed, because then the opportunistic labour inspectors would probably not change their behaviour.

Despite the dominance of the individualistic enforcement style, hierarchy was also very important in enforcement decisions. The hierarchical role of the public prosecutor was, for example, crucial in the decision-making processes in a few cases. Individual discretion was in these cases disregarded in favour of following orders from the public prosecutor, as a result of the importance mechanism. Also the supervisor sometimes managed to overpower the labour inspector's individual discretion, especially when labour inspectors were still in their internship. In other cases, hierarchical orders were disregarded, because applying them did not seem fair according to the labour inspectors.

These results should, however, be treated with caution, because this ethnographic study was not aimed at generalization towards a population but at theory-building. Another limitation of this study is that the causal mechanisms in the conceptual framework cannot be used to predict behaviour, but only to explain it afterwards. This is, however, not uncommon in social research, where prediction is rarely feasible (Elster, 1998). This paper rather aims at illustrating how GGCT can benefit from being supplemented with middle-range theories in the causal mechanism approach. Moreover, it aims to encourage future research in which both the conceptual framework and empirical findings in this study are tested.

\section{REFERENCES}

6, P. (2003), "Institutional Viability: a Neo-Durkheimian Theory", Innovation, Vol. 16 No. 4, pp. 395415.

Bakker, W. \& van Waarden, F. (1999), Ruimte rond regels. Stijlen van regulering en beleidsuitvoering vergeleken, Meppel, Boom: 396 p.

Bandura, A. (1990a), "Mechanisms of moral disengagement", in W. Reich (Ed.), Origins of terrorism: psychologies, ideologies, theologies, states of mind, Cambridge University Press, Cambridge, pp. 161-191.

Bandura, A. (1990b), "Selective activation and disengagement of moral control", Journal of Social Issues, Vol. 46 No. 1, pp. 27-46.

Bandura, A. (1999), "Moral disengagement in the perpetration of inhumanities", Personality and Social Psychology Review, Vol. 3 No. 3, pp. 193-209.

Bandura, A. (2002), "Social cognitive theory in cultural context", Applied Psychology: An International Review, Vol. 51 No. 2, pp. 269-290.

Biddle, B. J. (1986), "Recent developments in role theory", Annual Review of Sociology, Vol. 12 No. 1, pp. 67-92.

Brigham, J. \& Brown, D. W. (1980), Policy implementation: penalties or incentives, Sage, Beverly Hills: $271 \mathrm{p}$.

Davis, K. C. (1969), Discretionary justice: a preliminary inquiry, Louisiana State University Press, Baton Rouge: $233 \mathrm{p}$. 
Deutsch, K. (1985), "On theory and research in innovation", in R. Merrit \& A. Merrit (Eds.), Innovation in the public sector, Sage, Beverly Hills, pp. 17-35.

Diener, E. (1977), "Deindividuation: causes and consequences", Social behavior and personality, Vol. 5, pp. 143-156.

Douglas, M. \& Ney, S. (1998), Missing persons: A critique of the social sciences, University of California Press, Berkeley.

Douglas, M. (1982), In the active voice, Routledge Kegan \& Paul, London: 320 p.

Edwards, G. C. (1980), Implementing public policy, Congressional Quarterly Press, Washington DC.

Geertz, C. (1973), The interpretation of cultures, Fontana, New York.

George, A. L. \& Bennett, A. (2005), Case studies and theory development in the social sciences, MIT Press, Cambridge: $331 \mathrm{p}$.

Goode, W. J. (1960), "A theory of role strain", American Sociological Review, Vol. 25 No. 4, pp. 483496.

Gross, N., Mason, W., \& McEachern, A. (1958), Explorations in role analysis, Wiley, New York.

Hall, D. T. (1972), "A model of coping with role conflict: the role behavior of college educated women", Administrative Science Quarterly, Vol. 17 No. 4, pp. 471-486.

Hammersley, M. \& Atkinson, P. (1995), Ethnography. Principles in practice, Routledge, London: 323 p.

Handler, J. F. (1986), The conditions of discretion: autonomy, community, bureaucracy, Russell Sage Foundation, New York: $327 \mathrm{p}$.

Hedström, P. \& Swedberg, R. (1998), Social mechanisms. An analytical approach to social theory, Cambridge University Press, Cambridge: 340 p.

Hendriks, F. (1999), "The post-industrialising city: political perspectives and cultural biases", GeoJournal, Vol. 47 No. 3, pp. 425-432.

Hood, C. (1998), The art of the state. Culture, rhetoric, and public management, Clarendon Press, Oxford.

Jensen, L. (1998), "Cultural theory and democratizing functional domains. The case of Danish housing", Public administration, Vol. 76 No. 1, pp. 117-139.

Jervis, R. (1997), System effects: complexity in political and social life, Princeton University Press, Princeton.

Jowell, J. (1973), "The legal control of administrative discretion", Public Law, pp. 178-220.

Kast, F. E. \& Rosenzweig, J. E. (1972), "General systems theory: applications for organization and management", Academy of Management Journal, Vol. 15 No. 4, pp. 447-465.

Kelman, H. C. \& Hamilton, V. L. (1989), Crimes of obediende: toward a social psychology of authority and responsibility, Yale University Press, New Haven, CT.

Levinson, D. J. (1959), "Role, personality, and social structure in the organizational setting", Journal of Abnormal Psychology, Vol. 58 No. 2, pp. 170-180.

Lincoln, Y. (1985), Organization theory and inquiry: the paradigm revolution, Sage, Beverly Hills: 231 p.

Linder, S. \& Peters, B. G. (1987), "A design perspective on policy implementation: the fallacies of misplaced prescription", Policy Studies Review, Vol. 6 No. 3, pp. 459-476.

Lipsky, M. (1980), Street level bureaucracy: dilemmas of the individual in public services, Russell Sage Foundation, New York: $244 \mathrm{p}$. 
Loyens, K. (2012a), "Performance management or just counting numbers? Balancing conflicting demands in a West-European labor inspection", XVI IRSPM Conference, 11-13 April 2012,Rome (Italy).

Loyens, K. (2012b), "Towards a custom-made whistleblowing policy: Using grid-group cultural theory to match policy measures to different styles of peer reporting", Journal of Business Ethics (DOI 10.1007/s10551-012-1344-0).

Loyens, K. (2013), "Why police officers and labour inspectors (do not) blow the whistle: a grid group cultural theory perspective", Policing: an international journal of police strategies and management, Vol. 36 No. 1, pp. 27-50.

Maesschalck, J. (2004a), "Research note: a method for applying cultural theory in the study of organizations", Innovation: the European Journal of Social Sciences, Vol. 17 No. 4, pp. 377-386.

Maesschalck, J. (2004b), Towards a public administration theory on public servant's ethics, Katholieke Universiteit Leuven, Leuven.

Mars, G. (1982), Cheats at work. An anthropology of workplace crime, Ashgate, Aldershot.

Mayntz, R. (2004), "Mechanisms in the analysis of social macro-phenomena", Philosophy of the Social Sciences, Vol. 34 No. 2, pp. 237-259.

Merton, R. K. (1957), "The Role-Set: Problems in Sociological Theory", The British Journal of Sociology, Vol. 8 No. 2, pp. 106-120.

Moore, C. (2008), "Moral disengagement in processes of organizational corruption", Journal of Business Ethics, Vol. 80, pp. 129-139.

O'Riordan, T. \& Jordan, A. (1999), "Institutions, climate change and cultural theory: Towards a common analytical framework", Global Environmental Change, Vol. 9 No. 2, pp. 81-93.

Peterson, M. F. \& Smith, P. B. (2000), "Sources of meaning, organizations, and culture. Making sense of organizational events", in N. M. Ashkanasy, C. P. M. Wilderom, \& M. F. Peterson (Eds.), Handbook of organizational culture and climate, Sage Publications Inc., London, pp. 101-115.

Pollock, J. (1994), Ethics in crime and justice: dilemmas and decisions, Wadsworth, Belmont, CA.

Pollock, P. H., Lilie, S. A., \& Vittes, M. E. (1993), "Hard issues, core values and vertical constraint: The case of nuclear power", British Journal of Political Sciences, Vol. 23 No. 1, pp. 29-50.

Richards, L. \& Morse, J. M. (2007), Readme first for a user's guide to qualitative methods, Sage, Thousand Oaks (CA): $288 \mathrm{p}$.

Rogers, E. \& Joung-Imkim (1985), "Diffusion of innovation in public organizations", in R. Merrit \& A. Merrit (Eds.), Innovation in the public sector, Sage, Beverly Hills,

Rose, G. M., Kahle, L. R., \& Shokam, A. (2000), "Role relaxation and organizational culture. A social values perspectives", in N. M. Ashkanasy, C. P. M. Wilderom, \& M. F. Peterson (Eds.), Handbook of organizational culture and climate, Sage Publications Inc., London, pp. 437-446.

Sabatier, P. \& Mazmanian, D. (1979), "The conditions of effective implementation: a guide to accomplishing policy objectives", Policy Analysis, Vol. 5 No. 4, pp. 481-504.

Schwartz, B. (1991), "A pluralistic model of culture", Contemporary Sociology, Vol. 20 No. 5, pp. 764766.

Sieber, S. D. (1974), "Toward a theory of role accumulation", American Sociological Review, Vol. 39 No. 4, pp. 567-578.

Smaling, A. (2003), "Inductive, analogical, and communicative generalization", International Journal of Qualitative Methods, Vol. 2 No. 1, pp. 52-67. 
Stinchcombe, A. L. (1991), "The conditions of fruitfulness of theorizing about mechanisms in social science", Philosophy of the Social Sciences, Vol. 21 No. 3, pp. 367-388.

Thompson, M., Ellis, R., \& Wildavsky, A. (1990), Cultural Theory, Westview Press, Boulder.

Thompson, M. \& Rayner, S. (1998), "Risk and governance part I: The discourses of climate change", Government and Opposition, Vol. 33 No. 2, pp. 139-166.

Thompson, V. A. (1975), Without sympathy or enthusiasm: the problem of administrative compassion, University of Alabama Press, Alabama: $137 \mathrm{p}$.

Thornton, R. \& Nardi, P. M. (1975), "The dynamics of role acquisition", The American Journal of Sociology, Vol. 80 No. 4, pp. 870-885.

Verweij, M., Douglas, M., Ellis, R., Engel, C., Hendriks, F., Lohmann, S. et al. (2006), "Clumsy solutions for a complex world: The case of climate change", Public administration, Vol. 84 No. 4, pp. $817-$ 843.

Yin, R. K. (2003), Case study research. Design and methods (3rd ed.), Sage Publications, Thousand Oaks, CA. 